\title{
Feasibility of the sustainable EAT-Lancet diet for patients with Chronic Kidney Disease (CKD)
}

\author{
M. Borg ${ }^{1}$, L. Coneyworth ${ }^{1}$ and S. Welham ${ }^{1}$ \\ ${ }^{1}$ School of Biosciences, Faculty of Science, University of Nottingham, Sutton Bonington Campus, LE12 5RD, UK
}

Western diets contribute appreciably to suboptimal nutrition and environmental damage. The EAT-Lancet diet is a healthy, environmentally sustainable, predominantly plant based diet and could be a solution to both problems ${ }^{(1)}$. Dietary restrictions are often advised for patients with chronic kidney disease (CKD), especially in protein, potassium, and phosphate intake, to avoid comorbidities $^{(2)}$. No information is currently available regarding the suitability of the EAT-Lancet diet for this population. The aim of this study was therefore to evaluate if the EAT-Lancet diet can be recommended for CKD patients and assess the changes in nutrients from current dietary practices in these patients. As the majority of CKD patients are in older age categories with $46.2 \%$ of CKD stage $3-5$ being $>65$ years $^{(3)}$, we additionally set out to assess the similarity between diets consumed by older UK adults and EAT-Lancet to understand the feasibility of transition.

Elderly (>65 years) Nottingham residents living independently in the community were recruited as part of a pilot study exploring the impact of care requirements on micronutrient intake. Ten participants completed 3-day food diaries, which were extracted and analysed using Nutritics to establish the macro- and micro-nutrient content of each diary. The EAT- Lancet reference diet was also processed using Nutritics and protein, potassium and phosphorus concentrations compared with the CKD recommendations for these nutrients. Ethical approval was granted for the collection of dietary data by the University of Nottingham faculty of medicine and health sciences research ethics committee (Ref: 252-1902).

The elderly study participants consumed a median of $81.5 \mathrm{~g}$ day-1 protein ( $95 \% \mathrm{CI}: 57.38-95.53 \mathrm{~g}$ day-1) which was similar to that calculated for the EAT-Lancet diet of $89 \mathrm{~g}$ day-1. The intake of potassium at $2821.50 \mathrm{mg}$ day-1 (95\% CI: 1970.59-3309.70 g day-1) was a little lower (88.1\%) than the EAT-Lancet (3202 mg day-1) whilst phosphorus intake was considerably lower at $991.50 \mathrm{mg}$ day-1 (95\% CI: 599.30-1306.28 mg day-1) compared with $1791 \mathrm{mg}$ day-1 for the EAT- Lancet diet. It was apparent that the intakes of the study participants were within the recommended intake ranges for potassium and phosphorus of 2000-3000 mg day-1 and 600-1000 mg day-1 respectively but the EAT-Lancet diet exceeded these recommendations for both.

The EAT-Lancet diet appears to be a feasible option for CKD patients and provides superior nutrient content to what is being consumed in proxy groups. Although EAT-Lancet phosphate and potassium content was higher than CKD recommendations, these nutrients are mainly plant-based and stored as phytates that are less bioavailable. Culinary processing is also likely to reduce them to acceptable levels. More studies are required to support the use of the EAT-

\section{References}

1. Willett, W. et al. (2019) The Lancet 393(10170):447-492.

2. St-Jules, D. E., Goldfarb, D. S. and Sevick, M. A. (2016) J Ren Nutr 26(5):282-7.

3. Public Health England (2014) Chronic kidney disease prevalence model, [online] PHE publications gateway number: 2014386, Available at https://assets. publishing.service.gov.uk/government/uploads/system/uploads/attachment_data/file/612303/Chronickid neydiseaseCKDprevalencemodelbriefing.pdf 